\title{
Decomposing Contours into Curves of Different Families
}

\author{
Vincenzo Caglioti \\ Artificial Intelligence and Robotics Project, Dipartimento di Elettronica e \\ Informazione, Politecnico di Milano, P.za Leonardo da Vinci 32, 20133 Milano, Italy

\begin{abstract}
Many contour segmentation methods search for a decomposition into curves of a same family (as, e.g., the polygonal approximation presented in [1]). However, the contours of real objects often are constituted by curves of different families. In this paper an entropic criterion is presented for the decomposition of a given contour using curves of different families. The entropic criterion accounts both for the information cost associated to the use of complex curves and the uncertainty cost associated to the larger curve fitting error deriving from the use of simple curves. Experimental results on real images are reported to show the application of the criterion to two different curve families, namely the lienar segments and the circular arcs.
\end{abstract}

\section{Introduction}

Many contour segmentation methods decompose a contour into curves of the same family (see, e.g., [2], [4]). Saund [3] presented a scale-invariant criterion given by the ratio between the lenght of the fitted curvilinear arc and the mean distance between the arc and the points assigned to it. However, this criterion can not be applied to the case of overlapping curve families such as, e.g., the family of the line segments and the family of the circular arcs. In fact, no criterion based on the fitting error would prefer a line segment to the best fitting circular arc, since the latter curve is characterized by a smaller fitting error.

In this paper an entropic criterion is proposed for the decomposition of a set of points into curves belonging to different, but possibly overlapping, families. According to this criterion, the larger fitting error resulting from fitting a set of points to a simpler curve is traded for the lower information cost associated to the representation of the simpler curve. The main issue in this criterion concerns how to combine, in a nonparametric way, the information cost involved in representing a curve and the fitting error related to associating a set of points to the curve.

The determination of a globally optimum decomposition, according to the adopted criterion, would involve a combinatorially explosing complexity. Therefore a method is proposed to determine a suboptimal decomposition. The performance of the suboptimal method are demonstrated on contours extracted from real images.

In Sect. 2, the entropic criterion is introduced and the decomposition problem is formulated. In Sect. 3, the suboptimal method is presented. In Sect. 4, the 
performance of the suboptimal method on contours extracted from real images is illustrated. Section 5 contains some concluding remarks.

\section{Entropic criterion for contour decomposition}

Let us consider a set $\mathcal{P}$ of $N$ points $P_{i}(i=1, \ldots, N)$, which are known to lie within a rectangular planar region of dimensions $R \times R$. A decomposition of the point set $\mathcal{P}$ is characterized by a set $\mathcal{C}=\left\{C_{1}, \ldots, C_{n}\right\}$ of curves, and it specifies an assignment of any point $P_{i} \in \mathcal{P}$ to a curve $C_{j} \in \mathcal{C}$.

One way to represent the position of the points $P_{i} \in \mathcal{P}$ in the planar domain $R \times R$ up to a resolution $\delta r_{o}$ is to represent their coordinates $\left(x_{i}, y_{i}\right)$ directly: this involves an information quantity related to both the domain dimension $R$ and the resolution $\delta r_{o}$.

An alternative way to represent the position of the points is through the set $\mathcal{C}$ of curves: any point $P_{i} \in \mathcal{P}$ is assigned to one of the curves of $\mathcal{C}$. In this case the involved information includes: (i) the information needed to specify the curves $C_{j}$ with $j=1, \ldots n$, and, for any point $P_{i} \in \mathcal{P}$, (ii) the information needed to specify that the point $P_{i}$ is associated to the curve $C_{j}$ (and not, e.g., to other curves), and (iii) the information required to represent the coordinates of the point $P_{i}$ up to a resolution $\delta r_{o}$, once $P_{i}$ is known to be assigned to the curve $C_{j}$.

The proposed criterion is given by the difference between the information associated to representing the set of points through the assigned curves and the information associated to representing the coordinates of the points directly.

To represent the information quantity, the relative information content is used. In particular, suppose the a priori and the a posteriori distribution of a random variable $U$ have the same shape: if the standard deviations of the a priori and a posteriori distributions are, respectively, $A$ and $\delta u_{o}$, then the relative information content associated to $U$ is given by $H(U)=\ln \left(A / \delta u_{o}\right)$.

\subsection{Direct representation of contour points}

In this representation, the coordinates of the contour points are directly specified up to a resolution $\delta r_{o}$.

We chose to represent a point through its distance from a reference curve. In this way, we represent the "lateral" coordinate of the point, while neglecting to specify its "longitudinal" coordinate (i.e., the its coordinate along a direction which is locally parallel to the reference curve). Thus, the relative information content associated to representing a set of contour points is

$$
H_{d}=\sum_{i=1}^{N} \ln \frac{R}{\delta r_{o}}=N \ln \frac{R}{\delta r_{o}} .
$$




\subsection{Contour points represented through a set of curves}

To represent the contour points through a set of curves, first the curves are represented, and then the lateral coordinate of each contour point with respect to its assigned curve is specified up to a resolution $\delta r_{o}$.

Suppose that the decomposition of the contour is through a set of $n$ curves. Each curve is represented by a set of parameters. For instance, straight lines are represented by the polar parameters $\rho$ and $\theta$, which represent the distance of the origin from the line, and the angle formed by the $X$ axis and the normal to the line. A circular arc is represented by its radius and by the cartesian coordinates of the center of the circle $\left(r_{j}, x_{j}, y_{j}\right)$.

If both the a priori and the a posteriori distribution of the parameters of the curve $C_{j}$ are Gaussians, whith covariance matrixes, respectively, $\Lambda_{j o}$ and $\Lambda_{j}$, the relative information content of the curve representation is

$$
H\left(C_{j}\right)=(1 / 2) \ln \left(\left|\Lambda_{j o}\right| /\left|\Lambda_{j}\right|\right)
$$

Here $\Lambda_{j o}$ is relative the dimension $R$ of the image screen, while $\Lambda_{j}$ is relative to the parameters of the curve $C_{j}$ fitted to the contour points assigned to it.

Now that the parameters of the curves have been specified, the single contour points have to be represented. For each contour point, first the assigned curve has to be specified, and then the lateral coordinate, relative to the associated curve, must be provided. Suppose that $N_{j}$, out of the $N$ contour points, are associated to the curve $C_{j}$ : then the information quantity needed to specify the curve associated to a generic contour point $P_{i}$ is

$$
H_{i}=-\sum_{j=1}^{n} \frac{N_{j}}{N} \ln \frac{N_{j}}{N} .
$$

Once a contour point $P_{i}$ is known to be associated to a curve $C_{j}$, the the lateral dispersion of the point about the curve has to be represented (neglecting the "longitudinal" coordinate of the point) up to a resolution $\delta r_{0}$.

The a priori uncertainty of the position of the point $P_{i}$ is now related to the variance $\sigma_{j}^{2}$ of the lateral dispersion about the curve $C_{j}$ assigned to the point: $\sigma_{j}^{2}$ is estimated, e.g., by a least squares method. To specify the lateral coordinate of $P_{i}$ with an accuracy $\delta r_{o}$, the needed information quantity is

$$
H_{i j(i)}=\ln \left(\sigma_{j(i)} / \delta r_{o}\right)
$$

where $j(i)$ indicates the index of the curve assigned to the point $P_{i}$. For a given decomposition of the set of contour points, the relative information content is

$$
H_{c}=\sum_{j=1}^{n} H\left(C_{j}\right)+N \cdot H_{i}+\sum_{i=1}^{N} H_{i j(i)} .
$$




\subsection{Global criterion}

The global criterion proposed in this paper, is the difference between the relative information content of the curve representation of the contour points and the relative information content of their direct representation Notice that the parameter $\delta r_{o}$ vanishes from the global criterion.

$$
H=\frac{1}{2} \sum_{\cdot j=1}^{n} \ln \frac{\left|\Lambda_{j_{o}}\right|}{\left|\Lambda_{j}\right|}-\sum_{j=1}^{n} N_{j} \ln \frac{N_{j}}{N}+\sum_{i=1}^{N} \ln \frac{\sigma_{i j(i)}}{R}
$$

\subsection{Expression of the determinants}

First let us consider a straight line $C_{j}$ fitted to a set of points $\left\{P_{1}, \ldots, P_{N_{j}}\right\}$. Suppose that the least square estimate of the line parameters are $\left(\rho_{j}, \theta_{j}\right)$, which represent, respectively, the distance from the origin to the line and the orientation of its normal. Let $\bar{x}_{j}=\left(\sum_{i} x_{i}\right) / N_{j}, \bar{y}_{j}=\left(\sum_{i} y_{i}\right) / N_{j}$, and $L_{j}^{2}=\sum_{i}\left(\left(x_{i}-\right.\right.$ $\left.\left.\bar{x}_{j}\right) \sin \theta_{j}-\left(y_{i}-\bar{y}_{j}\right) \cos \theta_{j}\right)^{2} / N_{j}$. The a posteriori covariance matrix referred to an origin placed at the center $\left(\bar{x}_{j}, \bar{y}_{j}\right)$ of the line is:

$$
\Lambda_{j}=\frac{1}{N_{j}}\left[\begin{array}{cc}
\sigma^{2} & 0 \\
0 & \sigma^{2} / L_{j}^{2}
\end{array}\right]
$$

Its determinant is $\left|\Lambda_{j}\right|=\sigma^{4} /\left(N_{j}^{2} L_{j}^{2}\right)$. It is supposed $\left|\Lambda_{j o}\right|=R^{2}$.

Now let us consider a circular arc $C_{j}$ fitted to a set of points $P_{i}\left(i=1, \ldots, N_{j}\right)$ characterized by their polar coordinates $\left(\rho_{i}, \theta_{i}\right)$. (For the fitting of a circular arc to a set of points see [5].) Suppose that these polar coordinates are referred to an origin which coincides with the estimated center of the cirle. Let $\sigma^{2}$ be the variance of the lateral dispersion of the points about the circular arc $\left(\sigma^{2}\right.$ can be approximated by the mean squared distances of the points from the best fitting circle). The inverse of the a posteriori covariance matrix of the circle parameters is:

$$
\Lambda_{j}^{-1}=\frac{1}{\sigma^{2}}\left[\begin{array}{ccc}
N_{j} & \sum_{i} \cos \theta_{i} & \sum_{i} \sin \theta_{i} \\
\sum_{i} \cos \theta_{i} & \sum_{i} \cos ^{2} \theta_{i} & \sum_{i} \cos \theta_{i} \sin \theta_{i} \\
\sum_{i} \sin \theta_{i} & \sum_{i} \cos \theta_{i} \sin \theta_{i} & \sum_{i} \sin ^{2} \theta_{i}
\end{array}\right] .
$$

The form of $\left|\Lambda_{j}\right|$ is particularly simple if the reference frame is rotated such that $\sum_{i} \sin \left(2 \theta_{i}\right)=0$. In this case is

$$
\left|\Lambda_{j}\right|=\frac{\left(\sigma^{6} / N_{j}\right)}{\left(\sum_{i} \cos ^{2} \theta_{i}\right)\left(\sum_{i} \sin ^{2} \theta_{i}\right)-\left(\sum_{i} \cos ^{2} \theta_{i}\right)\left(\sum_{i} \sin \theta_{i}\right)^{2}-\left(\sum_{i} \sin ^{2} \theta_{i}\right)\left(\sum_{i} \cos \theta_{i}\right)^{2}}
$$

For the a priori covariance matix, it is assumed that $\left|\Lambda_{j o}\right|=R^{6}$. 


\section{Criterion minimization}

Finding the decomposition which minimizes the criterion (1) would require a time exponential in the number $N$ of points. Therefore in this work a suboptimal method is adopted. A set of threshold values are considered. For each threshold value, the following procedure is executed.

The contour is decomposed into curves of the broadest family (in our case, into the circular arcs) by means of an edge-following technique [2]. The considered threshold value is adopted to compare the distance between the currently analyzed point and the currently followed edge. After the decomposition, the entropic criterion is calculated and a series of local refinements are attempted.

A first local refinement, called merging, consists in substituting two neighboring curves by a single curve, by fitting one curve to the set of points associated to the two above curves. The curve may belong to any of the considered families. A second local refinement, called splitting consists in substituting a single curve with two curves belonging to any of the considered families.

First merging steps are attempted. After each refinement, the new criterion value is calculated and compared with the previous one. If the criterion value decreases, then a further refinement step is attempted on the curve obtained at the previous step. If the criterion value does not decrease, the situation previous to the currently attempted step is recovered, and splitting steps are iterated on the last modified contour segments, until the criterion value does not decrease anymore. Once the process ends, the same process is carried out starting from a different set of neighboring segments.

The above operations are carried out for each of the considered threshold values. Once all the threshold values of the set have been considered, the best decomposition is retained (among those obtained in the previous steps).

\section{Experimental results}

The suboptimal method described in Sect. 3 has been applied to the contours, extracted from real images, shown in Figs. 1a-2a. In our case the dimension of the image is $R=256$. The threshold values used in the process range from 1 to 6 pixel. The obtained contour decompositions are shown in Figs. 1b-2b. The obtained contour decomposition shown in Figs. $1 \mathrm{~b}$ and $2 \mathrm{~b}$ capture the correct decomposition of the model of the reproduced objects.

\section{Conclusions}

An information-theoretic criterion has been presented for driving the decomposition of contours into curves of different families. The criterion allows to evaluate both the fitting error, deriving from fitting a curve to a set of points, and the information cost involved in representing the fitted curve. The reported experimental results seem promising, in that they capture the correct contour 
decomposition of the objects present in the original images. Future work will address the introduction of further curve families, the search for better optimization strategies, and the investigation of the applicability of the criterion to the fitting of $3 \mathrm{D}$ data.

\section{References}

1. J.G.Dunham, - "Optimum uniform piecewise linear approximation of planar curves" - IEEE Transactions on Pattern Analysis and Machine Intelligence 8 (1986)

2. D.S.Chen, - "A data-driven intermediate level feature extraction algorithm" - IEEE Transactions on Pattern Analysis and Machine Intelligence 11 (1989)

3. E. Saund, - "Identifying salient circular arcs on curves" - GVGIP: Image Understanding 58 (1993)

4. B.K.Ray, K.S.Ray, - "A non-parametric sequential method for polygonal approximation of digital curves" - Pattern Recognition Letters 15 (1994)

5. S.Yi, R.M.Haralick, L.G.Shapiro, - "Error propagation in machine vision" - Machine Vision and Applications 7 (1994)

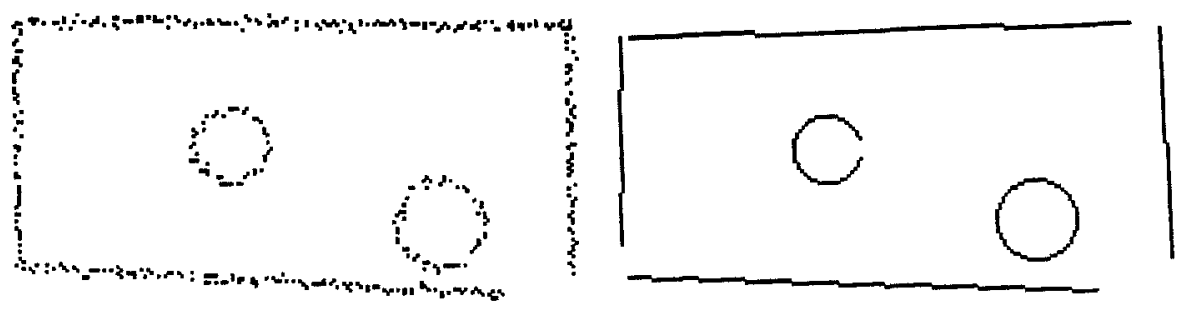

Fig. 1: A contour (a) and its decomposition (b)
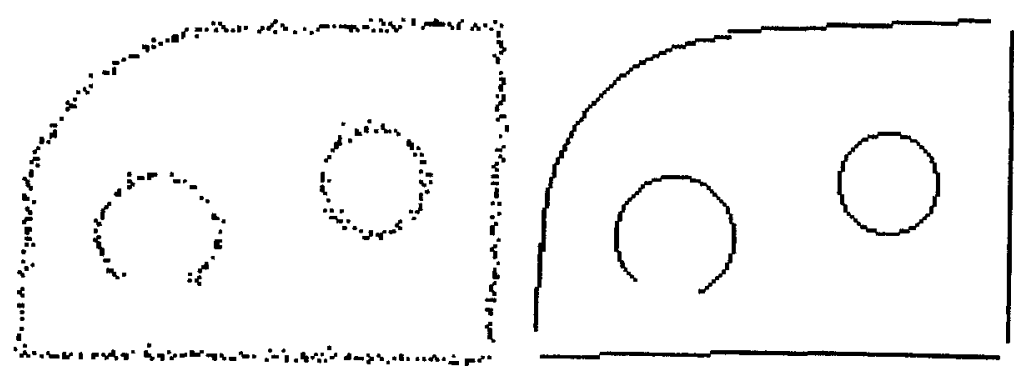

Fig. 2: A contour (a) and its decomposition (b) 